\title{
Hyers-Ulam and Hyers-Ulam-Rassias Stability for a Class of Integro-Differential Equations*
}

\author{
L. P. Castro ${ }^{\dagger}$ and A. M. Simões ${ }^{\ddagger}$
}

\subsection{Introduction}

The concept of stability for functional, differential, integral and integro-differential equations has been studied in a quite extensive way during the last six decades and have earned particular interest due to their great number of applications (see [1, 3, 5, 6, 8, 9, 10, 11, 12, 13, 14, 15, 16, 18, 19, 20, 21, 22, 23, 26] and the references therein). This occurs with particular emphasis in the case of Hyers-Ulam and HyersUlam-Rassias stabilities. These stabilities were originated from a famous question raised by S. M. Ulam at the University of Wisconsin in 1940: "When a solution of an equation differing slightly from a given one must be somehow near to the solution of the given equation?" A first partial answer to this question was given by D. H. Hyers, for Banach spaces, in the case of an additive Cauchy equation. This is why the obtained result is nowadays called the Hyers-Ulam stability. Different generalizations of that initial answer of D. H. Hyers were obtained by T. Aoki [2], Z. Gajda [17] and Th. M. Rassias [25]. The interested reader can obtain a detailed description of these advances in [4]. Afterwards, new directions were introduced by Th. M. Rassias, see [24], introducing therefore the so-called Hyers-Ulam-Rassias stability.

In this paper, we study the Hyers-Ulam stability and the Hyers-Ulam-Rassias stability for the following class of Volterra integro-differential equation,

\footnotetext{
*Accepted author's manuscript (AAM) of [Castro L.P., Simões A.M. (2019) Hyers-Ulam and Hyers-Ulam-Rassias Stability for a Class of Integro-Differential Equations. In: Taş K., Baleanu D., Machado J. (eds) Mathematical Methods in Engineering. Nonlinear Systems and Complexity, vol 23. Springer, Cham]. The final publication is available at Springer International Publishing AG, part of Springer Nature, via https://doi.org/10.1007/ 978-3-319-91065-9_3

TCenter for Research and Development in Mathematics and Applications (CIDMA), Department of Mathematics, University of Aveiro, Aveiro, Portugal; castro@ua.pt

${ }_{\ddagger}^{\ddagger}$ Center of Mathematics and Applications of University of Beira Interior (CMA-UBI), Department of Mathematics, University of Beira Interior, Covilhã, Portugal; asimoes@ubi.pt
} 


$$
y^{\prime}(x)=f\left(x, y(x), \int_{a}^{x} k(x, \tau, y(\tau), y(\alpha(\tau))) d \tau\right), \quad y(a)=c \in \mathbb{R},
$$

with $y \in C^{1}([a, b])$, for $x \in[a, b]$ where, for starting, $a$ and $b$ are fixed real numbers, $f:[a, b] \times \mathbb{C} \times \mathbb{C} \rightarrow \mathbb{C}$ and $k:[a, b] \times[a, b] \times \mathbb{C} \times \mathbb{C} \rightarrow \mathbb{C}$ are continuous functions, and $\alpha:[a, b] \rightarrow[a, b]$ is a continuous delay function (i.e., fulfilling $\alpha(\tau) \leq \tau$ for all $\tau \in[a, b])$.

The formal definition of the above mentioned stabilities are now introduced for our integro-differential equation (1.1).

If for each function $y$ satisfying

$$
\left|y^{\prime}(x)-f\left(x, y(x), \int_{a}^{x} k(x, \tau, y(\tau), y(\alpha(\tau))) d \tau\right)\right| \leq \theta, \quad x \in[a, b],
$$

where $\theta \geq 0$, there is a solution $y_{0}$ of the integro-differential equation and a constant $C>0$ independent of $y$ and $y_{0}$ such that

$$
\left|y(x)-y_{0}(x)\right| \leq C \theta,
$$

for all $x \in[a, b]$, then we say that the integro-differential equation $[1.1$ has the HyersUlam stability.

If for each function $y$ satisfying

$$
\left|y^{\prime}(x)-f\left(x, y(x), \int_{a}^{x} k(x, \tau, y(\tau), y(\alpha(\tau))) d \tau\right)\right| \leq \sigma(x), \quad x \in[a, b],
$$

where $\sigma$ is a non-negative function, there is a solution $y_{0}$ of the integro-differential equation and a constant $C>0$ independent of $y$ and $y_{0}$ such that

$$
\left|y(x)-y_{0}(x)\right| \leq C \sigma(x),
$$

for all $x \in[a, b]$, then we say that the integro-differential equation $(1.1)$ has the HyersUlam-Rassias stability.

Some of the present techniques to study the stability of functional equations use a combination of fixed point results with a generalized metric in appropriate settings. In view of this, and just for the sake of completeness, let us recall the definition of a generalized metric and the corresponding Banach Fixed Point Theorem.

Definition 1. Let $X$ a nonempty set. We say that a function $d: X \times X \rightarrow[0,+\infty]$ is a generalized metric on $X$ if:

1. $d(x, y)=0$ if and only if $x=y$;

2. $d(x, y)=d(y, x)$ for all $x, y \in X$;

3. $d(x, z) \leq d(x, y)+d(y, z)$ for all $x, y, z \in X$.

Theorem 1. Let $(X, d)$ be a generalized complete metric space and $T: X \rightarrow X$ a strictly contractive operator with a Lipschitz constant $L<1$. If there exists a nonnegative integer $k$ such that $d\left(T^{k+1} x, T^{k} x\right)<\infty$ for some $x \in X$, then the following three propositions hold true: 
1. the sequence $\left(T^{n} x\right)_{n \in \mathbb{N}}$ converges to a fixed point $x^{*}$ of $T$;

2. $x^{*}$ is the unique fixed point of $T$ in $X^{*}=\left\{y \in X: d\left(T^{k} x, y\right)<\infty\right\}$;

3. if $y \in X^{*}$, then

$$
d\left(y, x^{*}\right) \leq \frac{1}{1-L} d(T y, y)
$$

\subsection{Hyers-Ulam-Rassias Stability in the Finite Interval Case}

In this section we will present sufficient conditions for the Hyers-Ulam-Rassias stability of the integro-differential equation $[1.1$, where $x \in[a, b]$, for some fixed real numbers $a$ and $b$.

We will consider the space of continuously differentiable functions $C^{1}([a, b])$ on $[a, b]$ endowed with a generalization of the Bielecki metric

$$
d(u, v)=\sup _{x \in[a, b]} \frac{|u(x)-v(x)|}{\sigma(x)},
$$

where $\sigma$ is a non-decreasing continuous function $\sigma:[a, b] \rightarrow(0, \infty)$. We recall that $\left(C^{1}([a, b]), d\right)$ is a complete metric spaces (cf., [7], [27]).

Theorem 2. Let $\alpha:[a, b] \rightarrow[a, b]$ be a continuous delay function with $\alpha(t) \leq t$ for all $t \in[a, b]$ and $\sigma:[a, b] \rightarrow(0, \infty)$ a non-decreasing continuous function. In addition, suppose that there is $\beta \in[0,1)$ such that

$$
\int_{a}^{x} \sigma(\tau) d \tau \leq \beta \sigma(x)
$$

for all $x \in[a, b]$. Moreover, suppose that $f:[a, b] \times \mathbb{C} \times \mathbb{C} \rightarrow \mathbb{C}$ is a continuous function satisfying the Lipschitz condition

$$
|f(x, u(x), g(x))-f(x, v(x), h(x))| \leq M(|u(x)-v(x)|+|g(x)-h(x)|)
$$

with $M>0$ and the kernel $k:[a, b] \times[a, b] \times \mathbb{C} \times \mathbb{C} \rightarrow \mathbb{C}$ is a continuous function satisfying the Lipschitz condition

$$
|k(x, t, u(t), u(\alpha(t)))-k(x, t, v(t), v(\alpha(t)))| \leq L|u(\alpha(t))-v(\alpha(t))|
$$

with $L>0$.

$$
\begin{aligned}
& \text { If } y \in C^{1}([a, b]) \text { is such that } \\
& \qquad\left|y^{\prime}(x)-f\left(x, y(x), \int_{a}^{x} k(x, \tau, y(\tau), y(\alpha(\tau))) d \tau\right)\right| \leq \sigma(x), \quad x \in[a, b],
\end{aligned}
$$

and $M\left(\beta+L \beta^{2}\right)<1$, then there is a unique function $y_{0} \in C^{1}([a, b])$ such that

$$
y_{0}^{\prime}(x)=f\left(x, y_{0}(x), \int_{a}^{x} k\left(x, \tau, y_{0}(\tau), y_{0}(\alpha(\tau))\right) d \tau\right)
$$


and

$$
\left|y(x)-y_{0}(x)\right| \leq \frac{\beta}{1-M\left(\beta+L \beta^{2}\right)} \sigma(x)
$$

for all $x \in[a, b]$.

This means that under the above conditions, the integro-differential equation (1.1) has the Hyers-Ulam-Rassias stability.

Proof. By integration we have that

$$
y^{\prime}(x)=f\left(x, y(x), \int_{a}^{x} k(x, \tau, y(\tau), y(\alpha(\tau))) d \tau\right)
$$

is equivalent to

$$
y(x)=c+\int_{a}^{x} f\left(s, y(s), \int_{a}^{s} k(s, \tau, y(\tau), y(\alpha(\tau))) d \tau\right) d s .
$$

So, we will consider the operator $T: C^{1}([a, b]) \rightarrow C^{1}([a, b])$, defined by

$$
(T u)(x)=c+\int_{a}^{x} f\left(s, u(s), \int_{a}^{s} k(s, \tau, u(\tau), u(\alpha(\tau))) d \tau\right) d s,
$$

for all $x \in[a, b]$ and $u \in C^{1}([a, b])$.

Note that for any continuous function $u, T u$ is also continuous. Indeed,

$$
\begin{aligned}
\mid(T u)(x)- & (T u)\left(x_{0}\right) \mid \\
= & \mid \int_{a}^{x} f\left(s, u(s), \int_{a}^{s} k(s, \tau, u(\tau), u(\alpha(\tau))) d \tau\right) d s \\
& \quad-\int_{a}^{x_{0}} f\left(s, u(s), \int_{a}^{s} k(s, \tau, u(\tau), u(\alpha(\tau))) d \tau\right) d s \mid \\
= & \left|\int_{x}^{x_{0}} f\left(s, u(s), \int_{a}^{s} k(s, \tau, u(\tau), u(\alpha(\tau))) d \tau\right) d s\right| \rightarrow 0
\end{aligned}
$$

when $x \rightarrow x_{0}$.

Under the present conditions, we will deduce that the operator $T$ is strictly contractive with respect to the metric 1.7 . Indeed, for all $u, v \in C^{1}([a, b])$, we have,

$$
\begin{aligned}
& d(T u, T v)=\sup _{x \in[a, b]} \frac{|(T u)(x)-(T v)(x)|}{\sigma(x)} \\
& \leq M \sup _{x \in[a, b]} \frac{1}{\sigma(x)} \int_{a}^{x}|u(s)-v(s)| d s \\
& \quad+M \sup _{x \in[a, b]} \frac{1}{\sigma(x)} \int_{a}^{x} \int_{a}^{s}|k(s, \tau, u(\tau), u(\alpha(\tau)))-k(s, \tau, v(\tau), v(\alpha(\tau)))| d \tau d s
\end{aligned}
$$




$$
\begin{aligned}
\leq & M \sup _{x \in[a, b]} \frac{1}{\sigma(x)} \int_{a}^{x}|u(s)-v(s)| d s \\
& +M L \sup _{x \in[a, b]} \frac{1}{\sigma(x)} \int_{a}^{x} \int_{a}^{s}|u(\alpha(\tau))-v(\alpha(\tau))| d \tau d s \\
= & M \sup _{x \in[a, b]} \frac{1}{\sigma(x)} \int_{a}^{x} \sigma(s) \frac{|u(s)-v(s)|}{\sigma(s)} d s \\
& +M L \sup _{x \in[a, b]} \frac{1}{\sigma(x)} \int_{a}^{x} \int_{a}^{s} \sigma(\tau) \frac{|u(\alpha(\tau))-v(\alpha(\tau))|}{\sigma(\tau)} d \tau d s \\
\leq & M \sup _{s \in[a, b]} \frac{|u(s)-v(s)|}{\sigma(s)} \sup _{x \in[a, b]} \frac{1}{\sigma(x)} \int_{a}^{x} \sigma(s) d s \\
& +M L \sup _{\tau \in[a, b]} \frac{|u(\tau)-v(\tau)|}{\sigma(\tau)} \sup _{x \in[a, b]} \frac{1}{\sigma(x)} \int_{a}^{x} \int_{a}^{s} \sigma(\tau) d \tau d s \\
\leq & M d(u, v) \beta+M L d(u, v) \sup _{x \in[a, b]} \frac{\beta^{2} \sigma(x)}{\sigma(x)} \\
= & M\left(\beta+L \beta^{2}\right) d(u, v) .
\end{aligned}
$$

Due to the fact that $M\left(\beta+L \beta^{2}\right)<1$ it follows that $T$ is strictly contractive. Thus, we can apply the above mentioned Banach Fixed Point Theorem, which ensures that we have the Hyers-Ulam-Rassias stability for the integro-differential equation (1.1). Additionally, we can apply again the Banach Fixed Point Theorem, which guarantees us that

$$
d\left(y, y_{0}\right) \leq \frac{1}{1-M\left(\beta+L \beta^{2}\right)} d(T y, y) .
$$

From the definition of the metric $d, 1.8$ and 1.11 follows that

$$
\sup _{x \in[a, b]} \frac{\left|y(x)-y_{0}(x)\right|}{\sigma(x)} \leq \frac{\beta}{1-M\left(\beta+L \beta^{2}\right)}
$$

and consequently $(1.13)$ holds.

\subsection{Hyers-Ulam Stability in the Finite Interval Case}

In this section we will present sufficient conditions for the Hyers-Ulam stability of the integro-differential equation (1.1).

Theorem 3. Let $\alpha:[a, b] \rightarrow[a, b]$ be a continuous delay function with $\alpha(t) \leq t$ for all $t \in[a, b]$ and $\sigma:[a, b] \rightarrow(0, \infty)$ a non-decreasing continuous function. In addition, suppose that there is $\beta \in[0,1)$ such that

$$
\int_{a}^{x} \sigma(\tau) d \tau \leq \beta \sigma(x)
$$


for all $x \in[a, b]$. Moreover, suppose that $f:[a, b] \times \mathbb{C} \times \mathbb{C} \rightarrow \mathbb{C}$ is a continuous function satisfying the Lipschitz condition

$$
|f(x, u(x), g(x))-f(x, v(x), h(x))| \leq M(|u(x)-v(x)|+|g(x)-h(x)|)
$$

with $M>0$ and $k:[a, b] \times[a, b] \times \mathbb{C} \times \mathbb{C} \rightarrow \mathbb{C}$ is a continuous kernel function satisfying the Lipschitz condition

$$
|k(x, t, u(t), u(\alpha(t)))-k(x, t, v(t), v(\alpha(t)))| \leq L|u(\alpha(t))-v(\alpha(t))|
$$

with $L>0$.

If $y \in C^{1}([a, b])$ is such that

$$
\left|y^{\prime}(x)-f\left(x, y(x), \int_{a}^{x} k(x, \tau, y(\tau), y(\alpha(\tau))) d \tau\right)\right| \leq \theta, \quad x \in[a, b],
$$

where $\theta>0$ and $M\left(\beta+L \beta^{2}\right)<1$, then there is a unique function $y_{0} \in C^{1}([a, b])$ such that

$$
y_{0}^{\prime}(x)=f\left(x, y_{0}(x), \int_{a}^{x} k\left(x, t, y_{0}(t), y_{0}(\alpha(t))\right) d t\right)
$$

and

$$
\left|y(x)-y_{0}(x)\right| \leq \frac{(b-a) \sigma(b)}{\left[1-M\left(\beta+L \beta^{2}\right)\right] \sigma(a)} \theta
$$

for all $x \in[a, b]$.

This means that under the above conditions, the integro-differential equation (1.1) has the Hyers-Ulam stability.

This result can be obtained by using an analogous procedure as in the previous theorem (and so the full details of its proof are here omitted). In particular, we may consider the operator $T: C^{1}([a, b]) \rightarrow C^{1}([a, b])$, defined by

$$
(T u)(x)=c+\int_{a}^{x} f\left(s, u(s), \int_{a}^{s} k(s, \tau, u(\tau), u(\alpha(\tau))) d \tau\right) d s,
$$

for all $x \in[a, b]$ and $u \in C^{1}([a, b])$, and conclude that $T$ is strictly contractive with respect to the metric $(1.7)$, due to the fact that $M\left(\beta+L \beta^{2}\right)<1$. Thus, we can again apply the Banach Fixed Point Theorem, which in this case leads us to the HyersUlam stability for the integro-differential equation.

From 1.24) we have

$$
-\theta \leq y^{\prime}(x)-f\left(x, y(x), \int_{a}^{x} k(x, \tau, y(\tau), y(\alpha(\tau))) d \tau\right) \leq \theta, \quad x \in[a, b] .
$$

By integration in (1.28), we obtain 


$$
\left|y(x)-c-\int_{a}^{x} f\left(s, y(s), \int_{a}^{s} k(s, \tau, y(\tau), y(\alpha(\tau))) d \tau\right) d s\right| \leq \int_{a}^{x} \theta d \tau
$$

for all $x \in[a, b]$ and consequently

$$
|y(x)-(T y)(x)| \leq(b-a) \theta, \quad x \in[a, b] .
$$

By (1.6), the definition of the metric $d$ and 1.30 it is easy to prove the inequality (1.26).

\subsection{Hyers-Ulam-Rassias Stability in the Infinite Interval Case}

In this section, we analyse the Hyers-Ulam-Rassias stability of the integro-differential equation (1.1) but, instead of considering a finite interval $[a, b]$ (with $a, b \in \mathbb{R}$ ), we will consider the infinite interval $[a, \infty)$, for some fixed $a \in \mathbb{R}$.

Thus, we will now be dealing with the integro-differential equation

$$
y^{\prime}(x)=f\left(x, y(x), \int_{a}^{x} k(x, \tau, y(\tau), y(\alpha(\tau))) d \tau\right), \quad y(a)=c \in \mathbb{R}
$$

with $y \in C^{1}([a, \infty)), x \in[a, \infty)$ where $a$ is a fixed real number, $f:[a, \infty) \times \mathbb{C} \times \mathbb{C} \rightarrow \mathbb{C}$ and $k:[a, \infty) \times[a, \infty) \times \mathbb{C} \times \mathbb{C} \rightarrow \mathbb{C}$ are continuous functions, and $\alpha:[a, \infty) \rightarrow[a, \infty)$ is a continuous delay function which therefore fulfills $\alpha(\tau) \leq \tau$ for all $\tau \in[a, \infty)$.

Our strategy will be based on a recurrence procedure due to the already obtained result for the corresponding finite interval case.

Let us consider a fixed non-decreasing continuous function $\sigma:[a, \infty) \rightarrow(\varepsilon, \omega)$, for some $\varepsilon, \omega>0$ and the space $C_{b}^{1}([a, \infty))$ of bounded differentiable functions endowed with the metric

$$
d_{b}(u, v)=\sup _{x \in[a, \infty)} \frac{|u(x)-v(x)|}{\sigma(x)}
$$

Theorem 4. Let $\alpha:[a, \infty) \rightarrow[a, \infty)$ be a continuous delay function with $\alpha(t) \leq t$ for all $t \in[a, \infty)$ and $\sigma:[a, \infty) \rightarrow(\varepsilon, \omega)$, for some $\varepsilon, \omega>0$, a non-decreasing continuous function. In addition, suppose that there is $\beta \in[0,1)$ such that

$$
\int_{a}^{x} \sigma(\tau) d \tau \leq \beta \sigma(x)
$$

for all $x \in[a, \infty)$. Moreover, suppose that $f:[a, \infty) \times \mathbb{C} \times \mathbb{C} \rightarrow \mathbb{C}$ is a continuous function satisfying the Lipschitz condition

$$
|f(x, u(x), g(x))-f(x, v(x), h(x))| \leq M(|u(x)-v(x)|+|g(x)-h(x)|)
$$

with $M>0$ and the kernel $k:[a, \infty) \times[a, \infty) \times \mathbb{C} \times \mathbb{C} \rightarrow \mathbb{C}$ is a continuous function so that $\int_{a}^{x} k(x, \tau, z(\tau), z(\alpha(\tau))) d \tau$ is a bounded continuous function for any bounded continuous function $z$. In addition, suppose that $k$ satisfies the Lipschitz condition 


$$
|k(x, t, u(t), u(\alpha(t)))-k(x, t, v(t), v(\alpha(t)))| \leq L|u(\alpha(t))-v(\alpha(t))|
$$

with $L>0$.

If $y \in C_{b}^{1}([a, \infty))$ is such that

$$
\left|y^{\prime}(x)-f\left(x, y(x), \int_{a}^{x} k(x, \tau, y(\tau), y(\alpha(\tau))) d \tau\right)\right| \leq \sigma(x), \quad x \in[a, \infty),
$$

and $M\left(\beta+L \beta^{2}\right)<1$, then there is a unique function $y_{0} \in C_{b}^{1}([a, b])$ such that

$$
y_{0}^{\prime}(x)=f\left(x, y_{0}(x), \int_{a}^{x} k\left(x, \tau, y_{0}(\tau), y_{0}(\alpha(\tau))\right) d \tau\right)
$$

and

$$
\left|y(x)-y_{0}(x)\right| \leq \frac{\beta}{1-M\left(\beta+L \beta^{2}\right)} \sigma(x)
$$

for all $x \in[a, \infty)$.

This means that under the above conditions, the integro-differential equation (1.31) has the Hyers-Ulam-Rassias stability.

Proof. For any $n \in \mathbb{N}$, we will define $I_{n}=[a, a+n]$. By Theorem 2, there exists a unique bounded differentiable function $y_{0, n}: I_{n} \rightarrow \mathbb{C}$ such that

$$
y_{0, n}(x)=c+\int_{a}^{x} f\left(s, y_{0, n}(s), \int_{a}^{s} k\left(s, \tau, y_{0, n}(\tau), y_{0, n}(\alpha(\tau))\right) d \tau\right) d s
$$

and

$$
\left|y(x)-y_{0, n}(x)\right| \leq \frac{\beta}{1-M\left(\beta+L \beta^{2}\right)} \sigma(x)
$$

for all $x \in I_{n}$. The uniqueness of $y_{0, n}$ implies that if $x \in I_{n}$ then

$$
y_{0, n}(x)=y_{0, n+1}(x)=y_{0, n+2}(x)=\cdots .
$$

For any $x \in[a, \infty)$, let us define $n(x) \in \mathbb{N}$ as $n(x)=\min \left\{n \in \mathbb{N}: x \in I_{n}\right\}$. We also define a function $y_{0}:[a, \infty) \rightarrow \mathbb{C}$ by

$$
y_{0}(x)=y_{0, n(x)}(x) .
$$

For any $x_{1} \in[a, \infty)$, let $n_{1}=n\left(x_{1}\right)$. Then $x_{1} \in \operatorname{Int} I_{n_{1}+1}$ and there exists an $\varepsilon>0$ such that $y_{0}(x)=y_{0, n_{1}+1}(x)$ for all $x \in\left(x_{1}-\varepsilon, x_{1}+\varepsilon\right)$. By Theorem 2, $y_{0, n_{1}+1}$ is continuous at $x_{1}$, and so it is $y_{0}$.

Now, we will prove that $y_{0}$ satisfies

$$
y_{0}(x)=c+\int_{a}^{x} f\left(s, y_{0}(s), \int_{a}^{s} k\left(s, \tau, y_{0}(\tau), y_{0}(\alpha(\tau))\right) d \tau\right) d s
$$


and 1.38). For an arbitrary $x \in[a, \infty)$ we choose $n(x)$ such that $x \in I_{n(x)}$. By 1.39 and (1.42), we have

$$
\begin{aligned}
y_{0}(x) & =y_{0, n(x)}(x) \\
& =c+\int_{a}^{x} f\left(s, y_{0, n(x)}(s), \int_{a}^{s} k\left(s, \tau, y_{0, n(x)}(\tau), y_{0, n(x)}(\alpha(\tau))\right) d \tau\right) d s \\
& =c+\int_{a}^{x} f\left(s, y_{0}(s), \int_{a}^{s} k\left(s, \tau, y_{0}(\tau), y_{0}(\alpha(\tau))\right) d \tau\right) d s .
\end{aligned}
$$

Note that $n(\tau) \leq n(x)$, for any $\tau \in I_{n(x)}$, and it follows from 1.41 that $y_{0}(\tau)=$ $y_{0, n(\tau)}(\tau)=y_{0, n(x)}(\tau)$, so, the last equality in 1.44 holds.

To prove (1.38), by $(1.42)$ and $(1.40)$, we have that for all $x \in[a, \infty)$,

$$
\left|y(x)-y_{0}(x)\right|=\left|y(x)-y_{0, n(x)}(x)\right| \leq \frac{\beta}{1-M\left(\beta-L \beta^{2}\right)} \sigma(x) .
$$

Finally, we will prove the uniqueness of $y_{0}$. Let us consider another bounded differentiable function $y_{1}$ which satisfies (1.37] and 1.38, for all $x \in[a, \infty)$. By the uniqueness of the solution on $I_{n(x)}$ for any $n(x) \in \mathbb{N}$ we have that $y_{0 \mid I_{n(x)}}=y_{0, n(x)}$ and $y_{1 \mid I_{n(x)}}$ satisfies 1.37 and 1.38 for all $x \in I_{n(x)}$, so

$$
y_{0}(x)=\left.y_{0}\right|_{I_{n(x)}}(x)=\left.y_{1}\right|_{I_{n(x)}}(x)=y_{1}(x) .
$$

Remark 1 . With the necessary adaptations, Theorem 4 also holds true for infinite intervals of the type $(-\infty, b]$, with $b \in \mathbb{R}$, as well as for $(-\infty, \infty)$.

\subsection{Examples}

We will now present two concrete examples to illustrate the above presented results.

For a differentiable function $y:\left[0, \frac{2}{5}\right] \rightarrow \mathbb{R}$, let us consider the integro-differential equation

$$
y^{\prime}(x)=1+2 x-y(x)+\int_{0}^{x}\left(x(1+2 x) y(\tau) e^{\tau(x-\tau)}\right) d \tau, \quad x \in\left[0, \frac{2}{5}\right],
$$

as well as the continuous function $\sigma:\left[0, \frac{2}{5}\right] \rightarrow(0, \infty)$ defined by $\sigma(x)=3 e^{x}$ and the continuous delay function $\alpha:\left[0, \frac{2}{5}\right] \rightarrow\left[0, \frac{2}{5}\right]$ given by $\alpha(x)=x$.

We realize that all the conditions of Theorem 2 are here satisfied. In fact, $\alpha$ : $\left[0, \frac{2}{5}\right] \rightarrow\left[0, \frac{2}{5}\right]$ defined by $\alpha(x)=x$ is a continuous function with $\alpha(x) \leq x$. For $\beta=1 / 2$ we realize that $\sigma:\left[0, \frac{2}{5}\right] \rightarrow[0, \infty)$ defined by $\sigma(x)=3 e^{x}$, a continuous function, fulfills

$$
\int_{0}^{x} 3 e^{\tau} d \tau \leq \frac{3}{2} e^{x}=\beta \sigma(x), \quad x \in\left[0, \frac{2}{5}\right]
$$


Additionally $f:\left[0, \frac{2}{5}\right] \times \mathbb{C} \times \mathbb{C} \rightarrow \mathbb{C}$ defined by

$$
f(x, y(x), g(x))=1+2 x-y(x)+g(x)
$$

is a continuous function which satisfies

$$
|f(x, u(x), g(x))-f(x, v(x), h(x))| \leq|u(x)-v(x)|+|g(x)-h(x)|
$$

for all $x \in\left[0, \frac{2}{5}\right]$, and so we may take the constant $M$ considered in Theorem 2 to be equal to 1 .

The kernel $k:\left[0, \frac{2}{5}\right] \times\left[0, \frac{2}{5}\right] \times \mathbb{C} \rightarrow \mathbb{C}$ defined by

$$
k(x, t, y(t), y(\alpha(t)))=x(1+2 x) y(t) e^{t(x-t)}
$$

is a continuous function which fulfils the condition

$$
|k(x, t, u(t), u(\alpha(t)))-k(x, t, v(t), v(\alpha(t)))| \leq \frac{18}{25} e^{\frac{1}{25}}|u(\alpha(t))-v(\alpha(t))|
$$

for all $t \in[0, x]$ and $x \in\left[0, \frac{2}{5}\right]$, where we are using the constant $L=\frac{18}{25} e^{\frac{1}{25}}$. Thus, $M\left(\beta+L \beta^{2}\right)=\frac{1}{2}+\frac{9}{50} e^{1 / 25}<1$.

If we choose $y(x)=\frac{e^{x^{2}}}{0.3}$, it follows,

$$
\left|y^{\prime}(x)-f\left(x, y(x), \int_{a}^{x} k(x, \tau, y(\tau), y(\alpha(\tau))) d \tau\right)\right|=\left|\frac{7}{3}+\frac{14}{3} x\right| \leq \sigma(x),
$$

for all $x \in\left[0, \frac{2}{5}\right]$.

Therefore, this exhibits the Hyers-Ulam-Rassias stability of the integro-differential equation (1.47).

Moreover, by using the exact solution $y_{0}(x)=e^{x^{2}}$ we realize that

$$
\left|y(x)-y_{0}(x)\right|=\left|\frac{e^{x^{2}}}{0.3}-e^{x^{2}}\right| \leq \frac{3 e^{x}}{1-\frac{9}{25} e^{1 / 25}}=\frac{\beta}{1-M\left(\beta+L \beta^{2}\right)} \sigma(x)
$$

for all $x \in\left[0, \frac{2}{5}\right]$.

Let us now turn to a second example in which the Hyers-Ulam stability is illustrated.

For differentiable functions $y:[0,1] \rightarrow \mathbb{R}$, let us start be considering the integrodifferential equation

$$
y^{\prime}(x)=(-2 x-4) e^{x / 2}+5 y(x)+e^{x / 2} \int_{0}^{x}((\tau-x) y(\alpha(\tau))) d \tau,
$$

for all $x \in[0,1]$, as well as the continuous function $\sigma:[0,1] \rightarrow(0, \infty)$ defined by $\sigma(x)=1.1 e^{10 x}$ and the continuous delay function $\alpha:[0,1] \rightarrow[0,1 / 2]$ given by $\alpha(x)=x / 2$. 
We have all the conditions of Theorem 3 being satisfied. In fact, such $\alpha:[0,1] \rightarrow$ $[0,1 / 2]$ defined by $\alpha(x)=x / 2$ is a continuous function, and obviously $\alpha(x) \leq x$. Moreover, for $\beta=1 / 10$ we have that $\sigma:[0,1] \rightarrow(0, \infty)$ defined by $\sigma(x)=1.1 e^{10 x}$ is a continuous function fulfilling

$$
\int_{0}^{x} 1.1 e^{10 \tau} d \tau \leq \frac{1.1}{10} e^{10 x}=\beta \sigma(x), \quad x \in[0,1] .
$$

Additionally $f:[0,1] \times \mathbb{C} \times \mathbb{C} \rightarrow \mathbb{C}$ defined by

$$
f(x, y(x), g(x))=(-2 x-4) e^{x / 2}+5 y(x)+e^{x / 2} g(x)
$$

is a continuous function which fulfills

$$
|f(x, u(x), g(x))-f(x, v(x), h(x))| \leq 5(|u(x)-v(x)|+|g(x)-h(x)|)
$$

for all $x \in[0,1]$, and so the previous constant $M$ is here taking the value 5 .

The kernel $k:[0,1] \times[0,1] \times \mathbb{C} \rightarrow \mathbb{C}$ defined by

$$
k(x, t, y(t), y(\alpha(t)))=(t-x) y(\alpha(t))
$$

is a continuous function which fulfils the condition

$$
|k(x, t, u(t), u(\alpha(t)))-k(x, t, v(t), v(\alpha(t)))| \leq\left|u\left(\frac{t}{2}\right)-v\left(\frac{t}{2}\right)\right|
$$

for all $t \in[0, x]$ and $x \in[0,1]$. Where we may identify 1 as the constant previously denoted by $L$. Thus, $M\left(\beta+L \beta^{2}\right)=11 / 20<1$.

If we choose $y(x)=100 e^{x} / 99$, it follows

$$
\left|y^{\prime}(x)-f\left(x, y(x), \int_{a}^{x} k(x, \tau, y(\tau), y(\alpha(\tau))) d \tau\right)\right|=\left|\left(-\frac{2}{99} x-\frac{4}{99}\right) e^{x / 2}\right| \leq \theta \text { (1.61) }
$$

for all $x \in[0,1]$ and where we consider $\theta:=0.1$.

Therefore, from Theorem 3, we have the Hyers-Ulam stability of the integrodifferential equation 1.55 .

In particular, having in mind the exact solution $y_{0}(x)=e^{x}$ of 1.55 , it follows that

$$
\left|y(x)-y_{0}(x)\right|=\left|\frac{100}{99} e^{x}-e^{x}\right| \leq \frac{2}{9} e^{10 x}=\frac{(b-a) \sigma(b)}{\left[1-M\left(\beta+L \beta^{2}\right)\right] \sigma(a)} \theta
$$

for all $x \in[0,1]$.

Acknowledgement. This work was supported in part by FCT-Portuguese Foundation for Science and Technology through the Center for Research and Development in Mathematics and Applications (CIDMA) of University of Aveiro, within UID/MAT/04106/2013, and through the Center of Mathematics and Applications of University of Beira Interior (CMA-UBI), within project UID/MAT/00212/2013. 


\section{References}

1. Alsulami, H.H., Gülyaz, S., Karapinar, E., Erhan, I.M.: An Ulam stability result on quasib-metric-like spaces. Open Math. 14, 1087-1103 (2016)

2. Aoki, T.: On the stability of the linear transformation in Banach spaces. J. Math. Soc. Jpn. 2, 64-66 (1950)

3. Bahyrycz, A., Brzdęk, J., Jablońska, E., Malejki, R.: Ulam's stability of a generalization of the Fréchet functional equation. J. Math. Anal. Appl. 442(2), 537-553 (2016)

4. Brillouët-Belluot, N., Brzdȩk, J., Ciepliński, K.: On some recent developments in Ulam's type stability. Abstr. Appl. Anal. 2012, p. 41 (2012)

5. Brzdęk, J., Cădariu, L., Ciepliński, K.: Fixed point theory and the Ulam stability. J. Funct. Space. 2014, Article ID 829419, p. 16 (2014)

6. Burton, T.A.: Volterra Integral and Differential Equations. Elsevier, Amsterdam (2005)

7. Cădariu, L., Găvruţa, L., Găvruça, P.: Weighted space method for the stability of some nonlinear equations. Appl. Anal. Disc. Math. 6(1), 126-139 (2012)

8. Castro, L.P., Guerra, R.C.: Hyers-Ulam-Rassias stability of Volterra integral equations within weighted spaces. Lib. Math. (N.S.) 33(2), 21-35 (2013)

9. Castro, L.P., Ramos, A.: Hyers-Ulam-Rassias stability for a class of nonlinear Volterra integral equations. Banach J. Math. Anal. 3(1), 36-43 (2009)

10. Castro, L.P., Ramos, A.: Hyers-Ulam and Hyers-Ulam-Rassias stability of Volterra integral equations with a delay. In: Integral Methods in Science and Engineering 1, edited by M. E. Perez, Birkhäuser, Boston, 85-94 (2010)

11. Castro, L.P., Ramos, A.: Hyers-Ulam stability for a class of Fredholm integral equations. In: Mathematical Problems in Engineering Aerospace and Sciences ICNPAA 2010, Proceedings of the 8th International Conference of Mathematical Problems in Engineering, Aerospace and Science, 171-176 (2011)

12. Castro, L.P., Simões, A.M.: Hyers-Ulam and Hyers-Ulam-Rassias stability of a class of Hammerstein integral equations. AIP Conference Proceedings 1798:020036, p. 10 (2017)

13. Castro, L.P., Simões, A.M.: Hyers-Ulam and Hyers-Ulam-Rassias stability of a class of integral equations on finite intervals. In: CMMSE'17, Proceedings of the 17th International Conference on Computational and Mathematical Methods in Science and Engineering I-IV, 507-515 (2017)

14. Cho, Y.J., Park, C., Rassias, T.M., Saadati, R.: Stability of Functional Equations in Banach Algebras, Springer International Publishing, Switzerland (2015)

15. Du, W.-S.: A generalization of Diaz-Margolis's fixed point theorem and its application to the stability of generalized Volterra integral equations. J. Inequal. Appl. 2015:407, p. 15 (2015)

16. Forti, G.-L.: Hyers-Ulam stability of functional equations in several variables. Aequationes Math. 50, 143-190 (1995)

17. Gajda, Z.: On stability of additive mappings. Int. J. Math. Math. Sci. 14(3), 431-434 (1991)

18. Hassan, A.M., Karapinar, E., Alsulami, H.H.: Ulam-Hyers Stability for MKC Mappings via Fixed Point Theory. J. Funct. Space. 2016, p. 11 (2016)

19. Hyers, D.H.: On the stability of linear functional equation. Proc. Natl. Acad. Sci. 27(4), 222-224 (1941)

20. Hyers, D.H., Isac, G., Rassias, Th.M.: Stability of Functional Equations in Several Variables, Birkhäuser, Basel (1998)

21. Jung, S.-M.: Hyers-Ulam-Rassias Stability of Functional Equations in Mathematical Analysis. Hadronic Press, Palm Harbor (2001) 
22. Jung, S.-M., Şevgin, S., Şevli, H.: On the perturbation of Volterra integro-differential equations. Appl. Math. Lett. 26, 665-669 (2013)

23. Popa, D., Raşa, I.: On the best constant in Hyers-Ulam stability of some positive linear operators. J. Math. Anal. Appl. 412(1), 103-108 (2014)

24. Rassias, Th.M.: On the stability of the linear mapping in Banach spaces. Proc. Amer. Math. Soc. 72, 297-300 (1978)

25. Rassias, Th.M.: On a modified Hyers-Ulam sequence. J. Math. Anal. Appl. 158(1), 106$113(1991)$

26. Şevgin, S., Şevli, H.: Stability of a nonlinear Volterra integro-differential equation via a fixed point approach. J. Nonlinear Sci. Appl. 9, 200-207 (2016)

27. Tisdell, C.C., Zaidi, A.: Basic qualitative and quantitative results for solutions to nonlinear, dynamic equations on time scales with an application to economic modelling. Nonlinear Anal. 68(1), 3504-3524 (2008) 